\title{
S Research Square \\ S2 Alar Iliac Screw and S1 Pedicle Screw Fixation for the Treatment of Sacral Fractures : A Finite Element Study
}

Jianxiong Zheng

Southern Medical University Nanfang Hospital

Xiaoreng Feng

Southern Medical University Nanfang Hospital

Jie Xiang

Southern Medical University Nanfang Hospital

Fei Liu

Southern Medical University Nanfang Hospital

Frankie K L Leung

University of Hong Kong,Queen Mary Hospital

Bin Chen ( $\nabla$ chenbin1@smu.edu.cn )

Division of Orthopaedics and Traumatology, Department of Orthopaedics, Nanfang Hospital, Southern Medical University, Guangzhou, China https://orcid.org/0000-0001-8682-5043

\section{Research Article}

Keywords: S2-alar-iliac screw, sacral fracture, pelvic ring injury, sacropelvic fixation, biomechanics

Posted Date: August 6th, 2021

DOl: https://doi.org/10.21203/rs.3.rs-770173/v1

License: (a) This work is licensed under a Creative Commons Attribution 4.0 International License. Read Full License 


\section{Abstract}

Background: Five different sacral fracture fixation methods were compared using finite element (FE) analysis to study their biomechanical characteristics.

Methods: Denis type I sacral fractures were created by FE modeling. Five different fixation methods for the posterior pelvic ring were simulated: sacroiliac screw (SIS), lumbopelvic fixation (LPF), transiliac internal fixator (TIFI), S2-alar-iliac (S2AI) screw and S1 pedicle screw fixation (S2AI-S1), and S2AI screw and contralateral S1 pedicle screw fixation (S2AI-CS1). Four different loading methods were implemented in sequence to simulate the force in standing, flexion, right bending, and left twisting, respectively. Vertical stiffness, relative displacement, and change in relative displacement were recorded and analyzed.

Results: As predicted by the FE model, the vertical stiffness of the five groups in descending order were S2AI-S1, SIS, S2AI-CS1, LPF, TIFI. In terms of relative displacement, S2AI-S1 and S2AI-CS1 groups displayed a smaller mean relative displacement, although the S2AI-CS1 group exhibited greater displacement in the upper sacrum than the S2AI-S1 group. The SIS group displayed a moderate mean relative displacement, although the displacement of the upper sacrum was smaller than that of the S2AICS1 group. The LPF and TIFI groups displayed larger mean relative displacements. In terms of change in relative displacement, the TIFI and LPF groups displayed the greatest fluctuations in their motion, while the SIS, S2AI-S1, and S2AI-CS1 groups displayed smaller fluctuations.

Conclusion: Compared with SIS, unilateral LPF and TIFI, S2AI-S1 displayed the greatest biomechanical stability of the FE models and can be used as a new method for the treatment of sacral fractures. When the S1 pedicle screw insertion point on the affected side is damaged, S2AI-CS1 can be used as a good alternative to S2AI-S1.

\section{Background}

Sacroiliac screws (SIS) and lumbopelvic fixation (LPF) have been widely used clinically as two conventional methods for the treatment of posterior pelvic ring injuries, but both methods have limitations[1-4]. Sacroiliac screw placement with a residual displacement of $10 \mathrm{~mm}$ or more can endanger adjacent neural and vascular structures [1]. In addition, the reported incidence of sacroiliac screw loosening is as high as $17.3 \%$, and that of screw failure is $11.8 \%$ [2]. There are many postoperative complications in the clinical practice of LPF, such as incision infection, screw prominence, implant failure, and reoperation [3, 4]. Long-term fixation of the lumbar vertebrae is prone to lower back discomfort [4]. Transiliac internal fixator (TIFI) has the advantages of being minimally invasive and having low postoperative infection rate, although it still cannot prevent local skin discomfort caused by screw protrusion [5].

To reduce the problems caused by the protrusion of the iliac screws, the S2-alar-iliac (S2Al) technique has been proposed to replace the iliac screw in lumbosacropelvic fixation [6, 7]. The S2AI screw is $15 \mathrm{~mm}$ deeper than the iliac screw, which makes it less prominent [6]. Biomechanical testing of S2Al screw 
fixation resulted in comparable stability versus traditional iliac screw [8]. Furthermore, the S2AI screw technique has fewer postoperative complications than the iliac screw technique [9]. However, the S2AI screw technique is mainly used in spine-related operations $[10,11]$, and there are few studies $[12,13]$ that applied the S2AI screw technique to treat posterior pelvic ring injuries. In these studies [12,13], the lumbar spine was fixed, which would limit normal lumbosacral movement.

For Isler type I sacral fractures, the lumbosacral joint is considered to be preserved and therefore has a certain degree of stability [14].The purpose of surgery is only to resolve pelvic instability. We propose the S2Al screw and the ipsilateral S1 pedicle screw fixation (S2AI-S1) as a new internal fixation method to address the problems in LPF, such as high invasiveness to soft tissue, and those related to the fixation range [15]. This short-segment fixation method maintains the mobility of the lumbar spine and resolves concerns about adjacent segment disease. In addition, we propose another configuration that is to fix the S2Al screw with the contralateral S1 pedicle screw (S2AI-CS1). However, it is still unclear whether the new internal fixation methods can meet the requirements of biomechanical stability for the treatment of sacral fractures. Furthermore, the differences in biomechanical stability between new internal fixation methods and traditional fixation methods remain to be elucidated.

The aim of this study was to investigate the biomechanical stability of S2AI-S1, S2AI-CS1, SIS, LPF, and TIFI for the treatment of Denis type I sacral fractures through finite element (FE) analysis.

\section{Materials And Methods}

CT data of the lumbar spine and pelvis were collected from a healthy male volunteer (30 years old, 175 $\mathrm{cm}, 70 \mathrm{~kg}$, normal bone structure, no tumors, no deformities, no lumbar spine and pelvic structural damage). The CT imaging data were processed using Mimics 21.0 (Materialise, Belgium), Geomagic Studio 2013 (Geomagic, USA), and Solidworks 2017 (Dassault Systèmes, France) software to construct a model of the lumbar spine and complete pelvis. The interpelvic ligaments include iliolumbar ligament, anterior sacroiliac ligament, long posterior sacroiliac ligament (LPSL), short posterior sacroiliac ligament (SPSL), interosseous sacroiliac ligament, sacrospinous ligament, sacrotuberous ligament, superior pubic ligament, and arcuate pubic ligament were simulated as spring structures in a finite element analysis software, ANSYS 17.0 (ANSYS, USA). Values of the Young's modulus (MPa) and Poisson's ratio (u) of cortical bone, cancellous bone, articular cartilage, intervertebral and interpubic disc tissue, and titanium metal were derived from the literature $[16,17]$. The properties of ligaments are expressed in stiffness $(\mathrm{N} / \mathrm{mm})[18]$. The thicknesses of the cortical bone and endplates in the FE model were assumed to be 1.3 $\mathrm{mm}$ and $0.8 \mathrm{~mm}$, respectively [19]. The thickness of sacral cartilage and iliac cartilage in the sacroiliac joint were $1.8 \mathrm{~mm}$ and $0.9 \mathrm{~mm}$, respectively. The gap between the two cartilages was $0.3 \mathrm{~mm}$ [20] (Table 1). All the bony parts and implants were meshed using a 10-node tetrahedron element. 
Table 1

The material properties used in the finite element models

\begin{tabular}{|c|c|c|c|}
\hline Materials & Young's modulus(MPa) & Poisson's ratio(u) & Reference \\
\hline Titanium screw/rod/plate & 110000 & 0.3 & [16] \\
\hline Cortical bone(Pelvis) & 17000 & 0.3 & [17] \\
\hline Cancellous bone(Pelvis) & 100 & 0.2 & [17] \\
\hline Cortical bone(Lumbar) & 12000 & 0.3 & [17] \\
\hline Cancellous bone(Lumbar) & 100 & 0.2 & [17] \\
\hline Posterior bony elements & 3500 & 0.25 & [16] \\
\hline Articular cartilage & 10 & 0.4 & [16] \\
\hline Bony endplate & 1000 & 0.4 & [16] \\
\hline Cartilage endplate & 25 & 0.25 & [16] \\
\hline Nucleus pulposus & 1 & 0.499 & [16] \\
\hline Matrix of annulus fibrosus & 4.2 & 0.45 & [16] \\
\hline Fibers of annulus fibrosus & 450 & 0.3 & [16] \\
\hline Interpubic disc & 5 & 0.45 & [16] \\
\hline \multirow[t]{2}{*}{ Ligament } & Stiffness & Number of elements & Reference \\
\hline & $(\mathrm{N} / \mathrm{mm})$ & & \\
\hline Anterior sacroiliac ligament & 700 & $10 \times 2$ & [18] \\
\hline Posterior sacroiliac ligament (long) & 1000 & $4 \times 2$ & [18] \\
\hline Posterior sacroiliac ligament (short) & 400 & $10 \times 2$ & [18] \\
\hline Interosseous sacroiliac ligament & 2800 & $4 \times 2$ & [18] \\
\hline Sacrospinous ligament & 1400 & $5 \times 2$ & [18] \\
\hline Sacrotuberous ligament & 1500 & $5 \times 2$ & [18] \\
\hline Superior pubic ligament & 500 & $1 \times 1$ & [18] \\
\hline Accurate pubic ligament & 500 & $1 \times 1$ & [18] \\
\hline Iliolumbar ligament & 1000 & $4 \times 2$ & [18] \\
\hline
\end{tabular}

In the FE models, the contact condition between sacroiliac joint cartilage was defined as friction contact, and the friction coefficient was 0.015 [21]. Meanwhile, a friction coefficient of 0.3 was applied between the interaction surfaces of fractures [21]. The interfaces between the superior and inferior articular 
processes, between the plates/bars and the screws, and between the screws thread part and bone were modeled with a bonded contact.

Denis type I models (right side) were obtained respectively by grid line segmentation. The ligaments were injured as follows: Denis type I sacral fracture accompanied injuries of the LPSL and part of the SPSL. The anterior pelvic ring destruction resulted in injuries of the interpubic disc, superior pubic ligament, and arcuate pubic ligament.

The anterior pelvic ring was fixed with a plate, and the methods of fixing the posterior pelvic ring were as follows: in the SIS group, a half-thread hollow screw $(7.3 \mathrm{~mm} \times 75 \mathrm{~mm})$ was placed horizontally into the S1 segment. In the S2AI-S1 and S2AI-CS1 groups, the S2Al screw $(7.5 \mathrm{~mm} \times 80 \mathrm{~mm})$ was inserted according to the method in the literature [22], and then connected to the S1 pedicle screw with a rod (Fig. 1.a-b). Unilateral LPF was formed by connecting L4-L5 pedicle screws and an iliac screw (7.5 $\mathrm{mm} \times 80 \mathrm{~mm}$ ), and TIFI was formed by connecting iliac screws on both sides. In the FE model, the long screws close to the fracture line were defined as the main screws, while the others were secondary screws.

For the boundary condition, a standing posture with two legs was simulated: the acetabulum on both sides was fixed with 6 degrees of freedom. For the loading condition, a vertical force of $500 \mathrm{~N}$ was applied on the top surface of the L4 to simulate the upper body weight. According to spinal three-column theory, the upper endplate of L4 shares $85 \%$ of the load, and the bilateral superior articular facets of L4 share $15 \%$ of the load [16]. In addition, flexion was simulated by a $500 \mathrm{~N}$ vertical load and a $10 \mathrm{Nm}$ moment of forward sagittal direction [21]. Right bending was simulated by a $500 \mathrm{~N}$ vertical load and a 10 $\mathrm{Nm}$ moment of right lateral direction. Left twisting was simulated by a $500 \mathrm{~N}$ vertical load and a $10 \mathrm{Nm}$ moment of left horizontal rotation direction.

The vertical stiffness of each group was calculated based on the vertical displacement of the center point of the upper surface of $S 1$ (vertical stiffness $=500 \mathrm{~N} /$ vertical displacement). Four pairs of points (Fig. 2) on both sides of the fracture line were selected for calculation of the relative displacement (RD) of the fracture. The RD was used to evaluate the stability effect of each group of internal fixation on the sacral fracture model. The smaller the RD, the better the stability of the fixation. In addition, changes in the relative displacement caused by three types of motion with respect to standing were calculated, to evaluate the range of fluctuation in the relative displacement of motion conditions relative to standing conditions. (Additional file 1)

\section{Results}

\section{The mesh convergence study}

The number of elements used in the FE models of SIS group, LPF group, TIFI group, S2AI-S1 group and S2Al-CS1 group were 766,579, 772,157, 754,849, 751,032 and 757,516. A mesh convergence study was conducted and the appropriate mesh resolution for the FE model was determined from the influence of 
the maximum von Mises stress on bone. Doubling the number of elements in the FE models changed the maximum von Mises stress on the bone by $2.22 \%, 1.85 \%, 2.07 \%, 1.95 \%$ and $1.99 \%$ for the SIS, LPF, TIFI, S2AI-S1, and S2AI-CS1 groups, respectively. This indicated that the number of meshes in the FE models can meet the accuracy requirements of the analysis.

\section{The validation of the pelvic FE models}

Under $500 \mathrm{~N}$ vertical loads, the FE predicted maximum compressive displacement $(0.640$ to $1.136 \mathrm{~mm})$ of the intact pelvic model is consistent with the corresponding experiment-measured peak compressive displacements ( 0.973 to $1.550 \mathrm{~mm}$ ) reported by Comstock et al. [23] under the same loading condition. In addition, similar to the simulative conditions in the study by Lu et al. [19], a $6.5 \mathrm{~mm}$ sacroiliac screw was used to fix the Tile $C$ type pelvic ring injury. The average displacement of the observation site of the sacral wing edge was $1.5820 \mathrm{~mm}$, which was close to $2.0 \mathrm{~mm}$ reported in the literature. Under similar experimental conditions of the hip bones positioned upside down as reported by Dalstra et al.[24], the FE model predicted von Mises stresses (3.259 to $10.747 \mathrm{MPa}$ under $600 \mathrm{~N}$ loads ) of a hip-bone material were consistent with the corresponding experiment-measured von Mises stresses at the eight strain gages ( 0.712 to $7.641 \mathrm{MPa}$ under $600 \mathrm{~N}$ loads ).

\section{The vertical stiffness of the FE models}

The vertical displacement (Z-axis) of the center point of the upper surface of the $\mathrm{S} 1$ vertebral body in the SIS, LPF, TIFI, S2AI-S1, and S2AI-CS1 groups were $0.7119 \mathrm{~mm}, 0.9861 \mathrm{~mm}, 1.0694 \mathrm{~mm}, 0.6864 \mathrm{~mm}$, and $0.7491 \mathrm{~mm}$, respectively. According to the stiffness calculation formula, as predicted by the FE model, the vertical stiffness of the five groups in descending order were S2AI-S1, SIS, S2AI-CS1, LPF, and TIFI.

\section{The maximum von Mises stress in the implant and bone around the screw}

Under $500 \mathrm{~N}$ vertical loading, the maximum von Mises stresses of the implants in the SIS, LPF, TIFI, S2AIS1, and S2Al-CS1 groups were 165.770 MPa, 97.354 MPa, 65.580 MPa, 55.262 MPa, and 63.589 MPa, respectively. The maximum von Mises stress values of all the implants were less than the yield stress of titanium [25]. (Fig. 3.a-e). Under $500 \mathrm{~N}$ vertical stress, the maximum von Mises stresses of the bone around the main screw in the SIS, LPF, TIFI, S2AI-S1, and S2AI-CS1 groups were $31.955 \mathrm{MPa}, 26.810 \mathrm{MPa}$, 21.390 MPa, 26.695 MPa, and 36.839 MPa, respectively. In addition, the maximum von Mises stress of the bone around the secondary screw of the last four groups were $10.402 \mathrm{MPa}, 0.638 \mathrm{MPa}, 26.304 \mathrm{MPa}$, and $7.715 \mathrm{MPa}$, respectively. The maximum von Mises stress of the bone around the screws in all models was within the cortical bone but smaller than the yield strength of cortical bone [26].

\section{Relative displacement and change in relative displacement}

Using four different loading methods, the mean RD $(\mathrm{mm})$ of the five groups in descending order were TIFI, LPF, SIS, S2AI-CS1, and S2AI-S1 (except that the mean RD of LPF in flexion and twisting left was greater than for TIFI) (Fig. 4a-d). The RD of the LPF and TIFI groups displayed a decreasing trend from point 1 to point 3 , but an increasing trend from point 3 to point 4 . The RD of the SIS group exhibited an increasing 
trend from point 1 to point 4 . The RD of the S2AI-S1 and S2Al-CS1 groups displayed a decreasing trend from point 1 to point 2, but an increasing trend from point 2 to point 4 . The RD of the S2Al-CS1 group at point 1 was greater than that of the S2AI-S1 and SIS groups, while the RD at point 2 to point 4 was similar to that of the S2Al-S1 group but smaller than the SIS group. (Additional file 2-5)

Compared with standing, the change in relative displacement of the SIS, S2AI-S1, and S2AI-CS1 groups for three different movements fluctuated less. For the LPF group, the change in relative displacement during flexion was large, while in right bending and left twisting they were small. For the TIFI group, the change in relative displacement was larger in flexion and left twisting, while smaller in right bending (Table 2).

Table 2

The change in relative displacement of the five fixation methods

\begin{tabular}{|c|c|c|c|c|c|}
\hline \multirow[t]{2}{*}{ Motion } & \multirow[t]{2}{*}{ Fixation method } & \multicolumn{4}{|c|}{ Relative displacement changes (mm) } \\
\hline & & 1 & 2 & 3 & 4 \\
\hline \multirow[t]{5}{*}{ Flexion-standing } & LPF & 0.3670 & 0.3455 & 0.3373 & 0.3909 \\
\hline & TIFI & 0.2906 & 0.1348 & 0.0855 & 0.0630 \\
\hline & SIS & 0.0811 & 0.0291 & 0.0290 & 0.0722 \\
\hline & S2AI-S1 & 0.0492 & 0.0125 & 0.0245 & 0.0581 \\
\hline & S2Al-CS1 & 0.1308 & 0.0101 & 0.0423 & 0.0878 \\
\hline \multirow[t]{5}{*}{ Right bending- standing } & LPF & 0.1181 & 0.0693 & 0.0526 & 0.0761 \\
\hline & TIFI & 0.1583 & 0.1147 & 0.0981 & 0.1074 \\
\hline & SIS & 0.0165 & 0.0452 & 0.0624 & 0.0758 \\
\hline & S2Al-S1 & 0.0352 & 0.0101 & 0.0134 & 0.0257 \\
\hline & S2Al-CS1 & 0.0534 & 0.0015 & 0.0059 & 0.0223 \\
\hline \multirow{5}{*}{$\begin{array}{l}\text { Left twisting- } \\
\text { standing }\end{array}$} & LPF & 0.1015 & 0.0915 & 0.0911 & 0.1041 \\
\hline & TIFI & 0.3292 & 0.2684 & 0.2612 & 0.2001 \\
\hline & SIS & 0.0608 & 0.0659 & 0.0965 & 0.0758 \\
\hline & S2Al-S1 & 0.0255 & 0.0073 & 0.0201 & 0.0123 \\
\hline & S2Al-CS1 & 0.0418 & 0.0092 & 0.0208 & 0.0139 \\
\hline
\end{tabular}




\section{Discussion}

An unstable pelvic fracture is a severe injury that is the cause of high mortality and morbidity [27]. Due to complex local anatomy, unique biomechanics, and poor bone quality, the fixation of sacral fractures remains a challenge [28]. A key aspect of sacral fracture repair is sufficient stability to counterbalance translational and rotational forces in the vertical and horizontal directions [3]. In addition, to improve the quality of life of patients following surgery, sacral implants should also minimize adverse effects on patients. In the present study, static FE analysis methodology that is widely accepted by the community was used to evaluate the stability of five internal fixation methods on sacral fracture. The results of simulation show that the new internal fixation methods for the treatment of Denis type I sacral fractures can meet safety and stability requirements.

In a biomechanical study, Acklin et al. found that under Denis II sacral fracture model with vertical loading, the average axial stiffness of SIS was greater than that of unilateral LPF [29]. Although a Denis type I fracture model was used in the present study, the results of the simulation were consistent with those in the literature. In an FE analysis study, Song et al. found that unilateral LPF could not provide sufficient horizontal and rotational stability for patients with unilateral sacral fractures [30]. In the present study, the change in relative displacement of the unilateral LPF fluctuated greatly in flexion compared with standing, indicating that unilateral LPF is potentially unstable. In another study, Vigdorchik et al. found that the stiffness of the SIS was greater than the pedicle screw construct (namely TIFI) under the sacroiliac joint injury model [31]. Although a Denis type I fracture model was used, the stiffness of SIS which is greater than TIFI in our study was consistent with the result of Vigdorchik et al.

A higher maximum von Mises stress indicates that the model has a higher risk of implant failure [32]. According to the results of the simulation (except SIS), the LPF group displayed a greater risk of implant failure, occurring at the connection between the iliac screw and rod. This confirms previous clinical studies which found that implant failure usually manifests as disengagement of the screw to rod connector [33]. The TIFI and S2AI-CS1 groups displayed a moderate risk of implant failure at the shaft and junction of the head and main screw. The S2Al-S1 group exhibited a lower risk of implant failure which also occurred at the shaft and the junction of the head and secondary screw.

Higher stresses on the bone around the screw may lead to screw loosening and secondary fractures [21]. Based on the results of the simulation, the SIS group has a higher risk of screw loosening than the LPF group. In a cyclic loading experiment by Acklin et al., SIS was found to be more prone to loosening than LPF [29], consistent with our prediction. Furthermore, the main screws were predicted to have a greater risk of loosening than the secondary screws. For the main screws, the S2AI-CS1 and SIS groups had a higher risk of screw loosening, the S2AI-S1 and LPF groups displayed a moderate risk of screw loosening, while the TIFI group had a lower risk of screw loosening. For the secondary screws, the S1 pedicle screw in the S2Al-S1 group has a higher risk of loosening than other screws. Therefore, it is believed that the disadvantage of S2AI-S1 is a relatively increased risk of loosening of the secondary screw. When comparing the S2AI-S1 group with the S2AI-CS1 group, the ipsilateral S1 pedicle screw had a greater 
influence in reducing the maximum von Mises stress for the S2AI screw than the contralateral S1 pedicle screw.

According to the mean RD values, the overall stability of the five internal fixation methods in the $\mathrm{FE}$ models, in descending order are: S2AI-S1, S2AI-CS1, SIS, LPF, and TIFI. Considering that unilateral LPF and TIFI are insufficient for stability of the posterior pelvic ring, it is considered necessary to add a sacroiliac screw to unilateral LPF or TIFI to enhance stability, as demonstrated in previous studies [29, 34]. The RD of different points reveals that a fracture is more stable the closer it is to a screw (S2AI screw/Sacroiliac screw). This indicates that a single-segment S1 sacroiliac screw provides limited stability for the lower sacrum. A number of researchers have suggested adding an S2 sacroiliac screw for biplanar stability $[19,35]$. Since the S2Al-CS1 group had greater RD at point 1 and point 4 than the S2AlS1 group, the former was slightly less stable when used in sacral fractures than the latter. In addition, although the overall stability of the S2AI-CS1 group for sacral fractures was superior to that of SIS, the stability of the upper sacrum was slightly worse.

The change in relative displacement reveals the principal reason for the difference in stability. TIFI and unilateral LPF are methods that can achieve indirect stabilization of sacral fractures through a screw-rod system, while SIS, S2AI-S1, and S2AI-CS1 can provide direct fixation of sacral fractures using screws. According to the $\mathrm{AO}$ principles of fracture management, fixation of the fracture interface using screws could create a preload [36]. This preload would compress the fracture and prevent separation, while friction between the fracture surfaces and between the screw and bone would oppose displacement due to shear [36]. By comparing the relative changes in displacement for three types of motion, the TIFI and LPF groups displayed greater fluctuations during motion, while the SIS, S2AI-S1, and S2AI-CS1 groups exhibited only small fluctuations. This indicates that the SIS, S2AI-S1, and S2AI-CS1 groups can achieve absolute stability for the fracture, while the TIFI and LPF groups can only achieve relative stability.

This study confirmed the role of the new internal fixation in the stability of Denis I sacral fractures. The invasiveness of LPF and problems with the fixation range [15] (limitation of mobility of lumbar vertebrae, adjacent segment disease, and the need for implant removal) suggest that S2AI-S1 may be a beneficial method for internal fixation. Compared with TIFI, S2AI-S1 is not only expected to improve in biomechanical stability, but also eliminate the problem of screw protrusion. Although S2AI-S1 may not be as minimally invasive as SIS [37], the former has better biomechanical stability and a lower risk of screw loosening than the latter. Considering that S2AI-CS1 is slightly less stable in the upper sacrum and that the main screw has a higher risk of loosening, such fixation is not recommend as the first choice. When the S1 pedicle screw insertion point on the affected side is damaged, S2AI-CS1 can be used as a good alternative to S2AI-S1. Although the new internal fixation method can meet the safety and stability requirements, patients are advised to avoid premature weight bearing to prevent implant loosening and loss of reduction.

\section{Limitations}


Nevertheless, some potential limitations related to this study should be noted. First, the biomechanical results obtained through finite element analysis are inadequate to fully confirm the stability of S2Al-S1, which needs to be further verified by cyclic loading experiments on cadaver bone models. Second, the screw in this experiment used simplified processing to reduce the calculation time and analysis error due to stress concentration. However, to obtain accurate stress distribution on the screw and the bone around the screw, the screw thread should be considered in the FE model [38]. Third, in this study, only the main ligaments were simulated, but in practice, some other ligaments and muscles may still play a role [39]. Therefore, there may be some differences between our simulation data and cadaver bone model data. Finally, the present study used CT data from a normal male pelvis for development of the FE model, and no anatomical differences between individuals were considered.

\section{Conclusion}

Compared with SIS, unilateral LPF, and TIFI, S2AI-S1 displayed the best biomechanical stability of the FE models and can be used as a new method for the treatment of posterior pelvic ring injuries. When the $\mathrm{S} 1$ pedicle screw insertion point on the affected side is damaged, S2AI-CS1 can be used as a good alternative to S2AI-S1.

\section{Declarations}

\section{Ethics approval and consent to participate:}

This study was approved by the Ethics Committee of the Nanfang Hospital of Southern Medical University (NFEC-2019-256). Informed consent was obtained from the participant included in the study.

\section{Consent for publication:}

Not applicable

\section{Availability of data and materials:}

All data generated or analysed during this study are included in this published article and its supplementary information files

\section{Competing interests:}

The authors declare that they have no competing interests

\section{Funding:}


This work was supported by Clinical Research Startup Program of Southern Medical University by highlevel university construction funding of Guangdong provincial department of education (grant numbers:LC2019ZD001); the Clinical Research Program of Nanfang Hospital, Southern Medical University (grant numbers:2019CR016); the high-level scientific research project of Yangjiang People's Hospital (grant numbers :G2020006);

\section{Author contributions}

All authors contributed to the study conception. $\mathrm{BC}$ and $\mathrm{FKL}$ designed the experiments and revised the manuscript. JZ was in charge of implementing the project and the first draft of the manuscript. XF analyzed and interpreted the patient data. JX and FL performed material preparation, data collection. All authors read and approved the final manuscript.

\section{Acknowledgements:}

Not applicable

\section{References}

1. Reilly MC, Bono CM, Litkouhi B, Sirkin M, Behrens FF. The effect of sacral fracture malreduction on the safe placement of iliosacral screws. J ORTHOP TRAUMA. 2003;17(2):88-94. doi:10.1097/00005131-200302000-00002.

2. Kim JW, Oh CW, Oh JK, Kyung HS, Park KH, Yoon SD, Yoon SH. The incidence of and factors affecting iliosacral screw loosening in pelvic ring injury. Arch Orthop Trauma Surg. 2016;136(7):9217. doi:10.1007/s00402-016-2471-3.

3. Jones CB, Sietsema DL, Hoffmann MF. Can lumbopelvic fixation salvage unstable complex sacral fractures? Clin Orthop Relat Res. 2012;470(8):2132-41. doi:10.1007/s11999-012-2273-z.

4. Sagi HC, Militano U, Caron T, Lindvall E. A comprehensive analysis with minimum 1-year follow-up of vertically unstable transforaminal sacral fractures treated with triangular osteosynthesis. J ORTHOP TRAUMA. 2009;23(5):313-9. doi:10.1097/BOT.0b013e3181a32b91. 319-321.

5. Dienstknecht T, Berner A, Lenich A, Nerlich M, Fuechtmeier B. A minimally invasive stabilizing system for dorsal pelvic ring injuries. Clin Orthop Relat Res. 2011;469(11):3209-17. doi:10.1007/s11999011-1922-y.

6. Chang TL, Sponseller PD, Kebaish KM, Fishman EK. Low profile pelvic fixation: anatomic parameters for sacral alar-iliac fixation versus traditional iliac fixation. Spine (Phila Pa 1976). 2009;34(5):43640. doi:10.1097/BRS.0b013e318194128c.

7. Sponseller PD, Zimmerman RM, Ko PS, Pull TGA, Mohamed AS, Chang TL, Kebaish KM. Low profile pelvic fixation with the sacral alar iliac technique in the pediatric population improves results at two- 
year minimum follow-up. Spine (Phila Pa 1976). 2010;35(20):1887-92.

doi:10.1097/BRS.0b013e3181e03881.

8. Hoernschemeyer DG, Pashuck TD, Pfeiffer FM. Analysis of the s2 alar-iliac screw as compared with the traditional iliac screw: does it increase stability with sacroiliac fixation of the spine? SPINE J. 2017;17(6):875-9. doi:10.1016/j.spinee.2017.02.001.

9. Hasan MY, Liu G, Wong HK, Tan JH. Postoperative complications of S2Al versus iliac screw in spinopelvic fixation: a meta-analysis and recent trends review. SPINE J. 2020;20(6):964-72. doi:10.1016/j.spinee.2019.11.014.

10. Unoki E, Miyakoshi N, Abe E, Kobayashi T, Abe T, Kudo D, Shimada Y. Sacropelvic Fixation With S2 Alar Iliac Screws May Prevent Sacroiliac Joint Pain After Multisegment Spinal Fusion. Spine (Phila Pa 1976). 2019;44(17):E1024-30. doi:10.1097/BRS.0000000000003041.

11. Ishida W, Ramhmdani S, Casaos J, Perdomo-Pantoja A, Elder BD, Theodore N, Gokaslan ZL, Wolinsky JP, Sciubba DM, Bydon A, et al. Safety Profile of Lumbosacropelvic Fixation in Patients Aged 60 Years or Older: Comparison Between S2-Alar-lliac Screws and Conventional Iliac Screws. CLIN SPINE SURG. 2019;32(4):E200-5. doi:10.1097/BSD.0000000000000806.

12. El DM, Shafiq B, Vaswani R, Osgood GM, Hasenboehler EA, Kebaish KM. Minimally Invasive Fixation for Spinopelvic Dissociation: Percutaneous Triangular Osteosynthesis with S2 Alar-lliac and Iliosacral Screws: A Case Report. JBJS Case Connect. 2019;9(4):e119. doi:10.2106/JBJS.CC.19.00119.

13. Martin CT, Witham TF, Kebaish KM. Sacropelvic fixation: two case reports of a new percutaneous technique. Spine (Phila Pa 1976). 2011;36(9):E618-21. doi:10.1097/BRS.0b013e3181f79aba.

14. Beckmann NM, Chinapuvvula NR. Sacral fractures: classification and management. Emerg Radiol. 2017;24(6):605-17. doi:10.1007/s10140-017-1533-3.

15. Futamura K, Baba T, Mogami A, Morohashi I, Obayashi O, Iwase H, Kaneko K. A biomechanical study of sacroiliac rod fixation for unstable pelvic ring injuries: verification of the "within ring" concept. INT ORTHOP. 2018;42(4):909-14. doi:10.1007/s00264-017-3713-x.

16. Li Y, He D, Chen N, Lv T, Wu A, Lin Z, Ding Z, Wang Z, Wu L. Optimal axis for lumbosacral interbody fusion: Prospective finite element analysis and retrospective 3D-CT measurement. CLIN ANAT. 2019;32(3):337-47. doi:10.1002/ca.23316.

17. Kim YH, Yao Z, Kim K, Park WM. Quantitative investigation of ligament strains during physical tests for sacroiliac joint pain using finite element analysis. Man Ther. 2014;19(3):235-41. doi:10.1016/j.math.2013.11.003.

18. Sohn S, Park TH, Chung CK, Kim YJ, Jang JW, Han IB, Lee SJ. Biomechanical characterization of three iliac screw fixation techniques: A finite element study. J CLIN NEUROSCI. 2018;52:109-14. doi:10.1016/j.jocn.2018.03.002.

19. Lu Y, He Y, Li W, Yang Z, Peng R, Yu L: Comparison of Biomechanical Performance of Five Different Treatment Approaches for Fixing Posterior Pelvic Ring Injury. J HEALTHC ENG 2020, 2020:5379593.doi:10.1155/2020/5379593. 
20. Li J, Peng Y, Yuchi C, Du C. [Finite element analysis of fixation of U-shaped sacral fractures]. Sheng Wu Yi Xue Gong Cheng Xue Za Zhi. 2019;36(2):223-31. doi:10.7507/1001-5515.201808026.

21. Hu P, Wu T, Wang HZ, Qi XZ, Yao J, Cheng XD, Chen W, Zhang YZ. Biomechanical Comparison of Three Internal Fixation Techniques for Stabilizing Posterior Pelvic Ring Disruption: A 3D Finite Element Analysis. ORTHOP SURG. 2019;11(2):195-203. doi:10.1111/os.12431.

22. Zhu F, Bao HD, Yuan S, Wang B, Qiao J, Zhu ZZ, Liu Z, Ding YT, Qiu Y. Posterior second sacral alar iliac screw insertion: anatomic study in a Chinese population. EUR SPINE J. 2013;22(7):1683-9. doi:10.1007/s00586-013-2734-4.

23. Comstock CP, van der Meulen MC, Goodman SB. Biomechanical comparison of posterior internal fixation techniques for unstable pelvic fractures. J ORTHOP TRAUMA. 1996;10(8):517-22. doi:10.1097/00005131-199611000-00001.

24. Dalstra M, Huiskes R, van Erning L. Development and validation of a three-dimensional finite element model of the pelvic bone. J Biomech Eng. 1995;117(3):272-8. doi:10.1115/1.2794181.

25. Zhang E, Li S, Ren J, Zhang L, Han Y. Effect of extrusion processing on the microstructure, mechanical properties, biocorrosion properties and antibacterial properties of Ti-Cu sintered alloys. Mater Sci Eng C Mater Biol Appl. 2016;69:760-8. doi:10.1016/j.msec.2016.07.051.

26. Li Z, Kim JE, Davidson JS, Etheridge BS, Alonso JE, Eberhardt AW. Biomechanical response of the pubic symphysis in lateral pelvic impacts: a finite element study. J BIOMECH. 2007;40(12):2758-66. doi:10.1016/j.jbiomech.2007.01.023.

27. Hao T, Changwei Y, Qiulin Z. Treatment of posterior pelvic ring injuries with minimally invasive percutaneous plate osteosynthesis. INT ORTHOP. 2009;33(5):1435-9. doi:10.1007/s00264-0090756-7.

28. Moshirfar A, Rand FF, Sponseller PD, Parazin SJ, Khanna AJ, Kebaish KM, Stinson JT, Riley LR. Pelvic fixation in spine surgery. Historical overview, indications, biomechanical relevance, and current techniques. J BONE JOINT SURG AM. 2005;87(Suppl 2):89-106. doi:10.2106/JBJS.E.00453.

29. Acklin YP, Zderic I, Richards RG, Schmitz P, Gueorguiev B, Grechenig S. Biomechanical investigation of four different fixation techniques in sacrum Denis type II fracture with low bone mineral density. $J$ ORTHOP RES. 2018;36(6):1624-9. doi:10.1002/jor.23798.

30. Song W, Zhou D, He Y. The biomechanical advantages of bilateral lumbo-iliac fixation in unilateral comminuted sacral fractures without sacroiliac screw safe channel: A finite element analysis. Medicine. 2016;95(40):e5026. doi:10.1097/MD.0000000000005026.

31. Vigdorchik JM, Jin X, Sethi A, Herzog DT, Oliphant BW, Yang KH, Vaidya R. A biomechanical study of standard posterior pelvic ring fixation versus a posterior pedicle screw construct. INJURY. 2015;46(8):1491-6. doi:10.1016/j.injury.2015.04.038.

32. Wu T, Ren X, Cui Y, Cheng X, Peng S, Hou Z, Han Y. Biomechanical study of three kinds of internal fixation for the treatment of sacroiliac joint disruption using biomechanical test and finite element analysis. J ORTHOP SURG RES. 2018;13(1):152. doi:10.1186/s13018-018-0858-2. 
33. Shabtai L, Andras LM, Portman M, Harris LR, Choi PD, Tolo VT, Skaggs DL. Sacral Alar lliac (SAI) Screws Fail 75\% Less Frequently Than Iliac Screws in Neuromuscular Scoliosis. J Pediatr Orthop. 2017;37(8):e470-5. doi:10.1097/BP0.0000000000000720.

34. Chaiyamongkol W, Kritsaneephaiboon A, Bintachitt P, Suwannaphisit S, Tangtrakulwanich B. Biomechanical Study of Posterior Pelvic Fixations in Vertically Unstable Sacral Fractures: An Alternative to Triangular Osteosynthesis. Asian Spine J. 2018;12(6):967-72. doi:10.31616/asj.2018.12.6.967.

35. Zhao Y, Li J, Wang D, Liu Y, Tan J, Zhang S. Comparison of stability of two kinds of sacro-iliac screws in the fixation of bilateral sacral fractures in a finite element model. INJURY. 2012;43(4):4904. doi:10.1016/j.injury.2011.12.023.

36. Buckley RE, Moran CG, Apivatthakakul T. AO Principles of Fracture Management. 3rd ed. Stuttgart: George Thieme Verlag; 2018.

37. Coste C, Asloum Y, Marcheix PS, Dijoux P, Charissoux JL, Mabit C. Percutaneous iliosacral screw fixation in unstable pelvic ring lesions: the interest of O-ARM CT-guided navigation. Orthop Traumatol Surg Res. 2013;99(4 Suppl):273-8. doi:10.1016/j.otsr.2013.03.002.

38. Shin JK, Lim BY, Goh TS, Son SM, Kim HS, Lee JS, Lee CS. Effect of the screw type (S2-alar-iliac and iliac), screw length, and screw head angle on the risk of screw and adjacent bone failures after a spinopelvic fixation technique: A finite element analysis. PLOS ONE. 2018;13(8):e201801. doi:10.1371/journal.pone.0201801.

39. Li J, Lu Y, Miller SC, Jin Z, Hua X. Development of a finite element musculoskeletal model with the ability to predict contractions of three-dimensional muscles. J BIOMECH. 2019;94:230-4. doi:10.1016/j.jbiomech.2019.07.042.

\section{Figures}
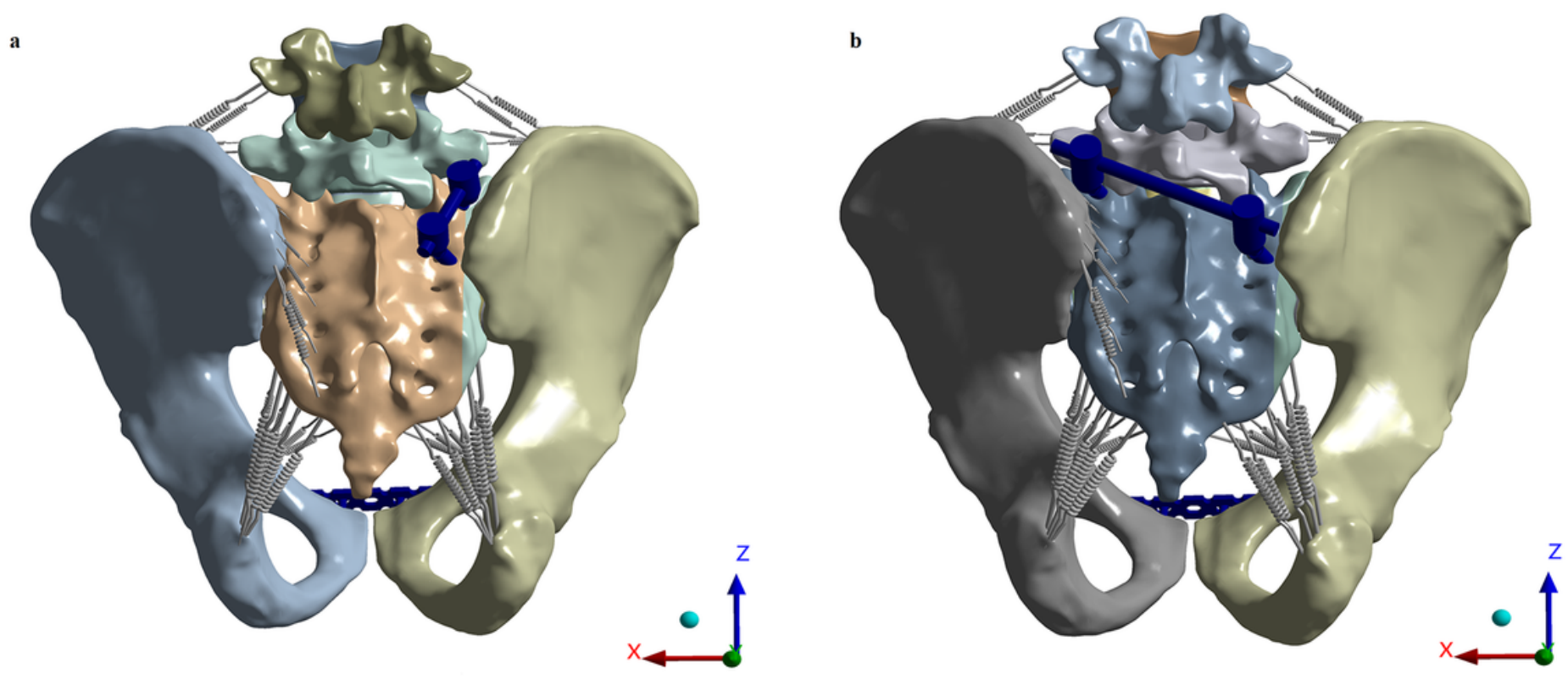


\section{Figure 1}

The FE models of new internal fixation method. a. S2Al-S1 model. b.S2Al-CS1 model.

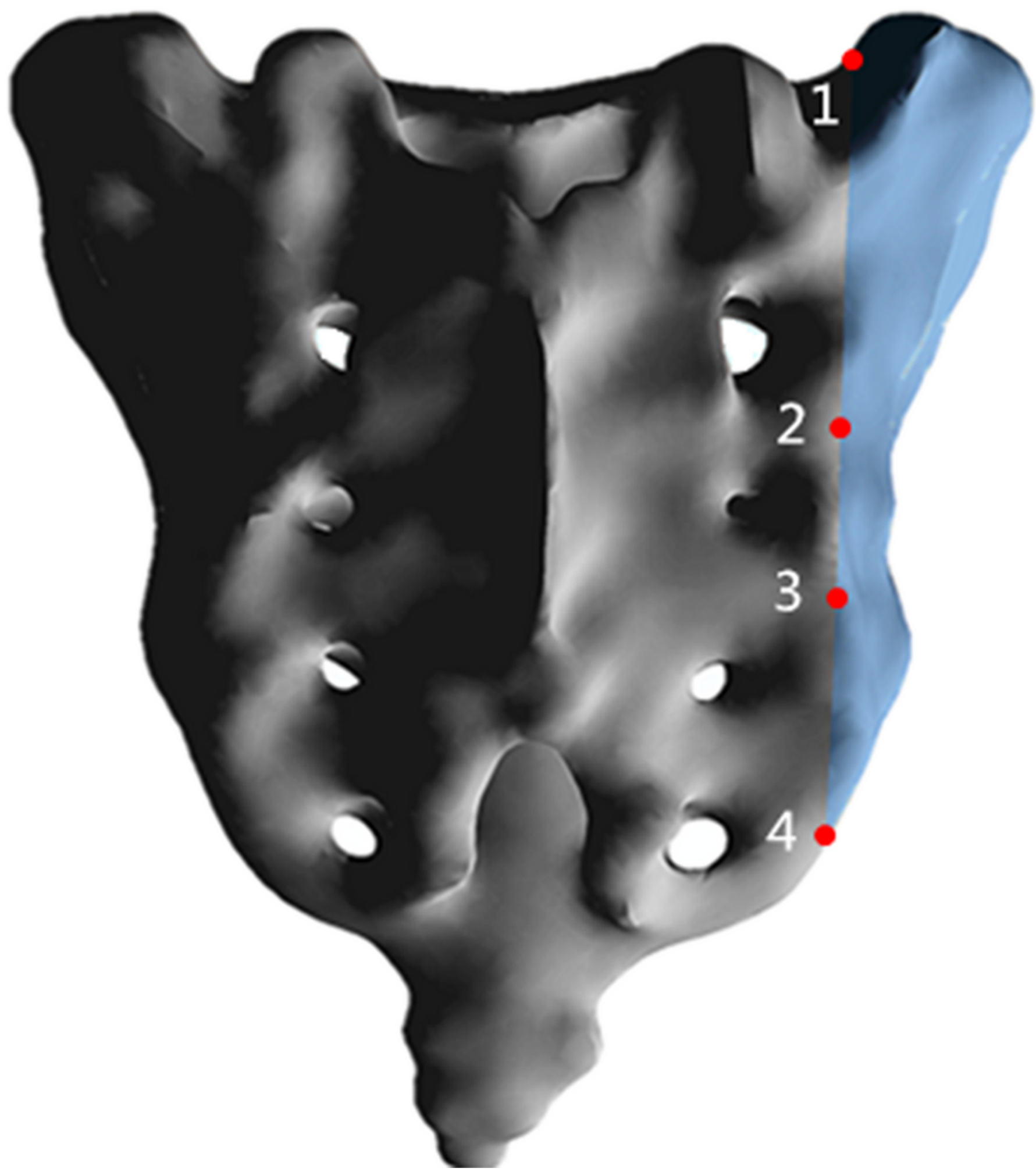

\section{Figure 2}

Four observation points in the FE model. Points 1 and 4 were located at the upper and lower ends of the sacrum, respectively. Point 2 was located at the intersection of the horizontal midline between S1 and S2 
posterior sacral foramen and the fracture line. Point 3 was located at the intersection of the horizontal midline between S2 and S3 posterior sacral foramen and the fracture line.
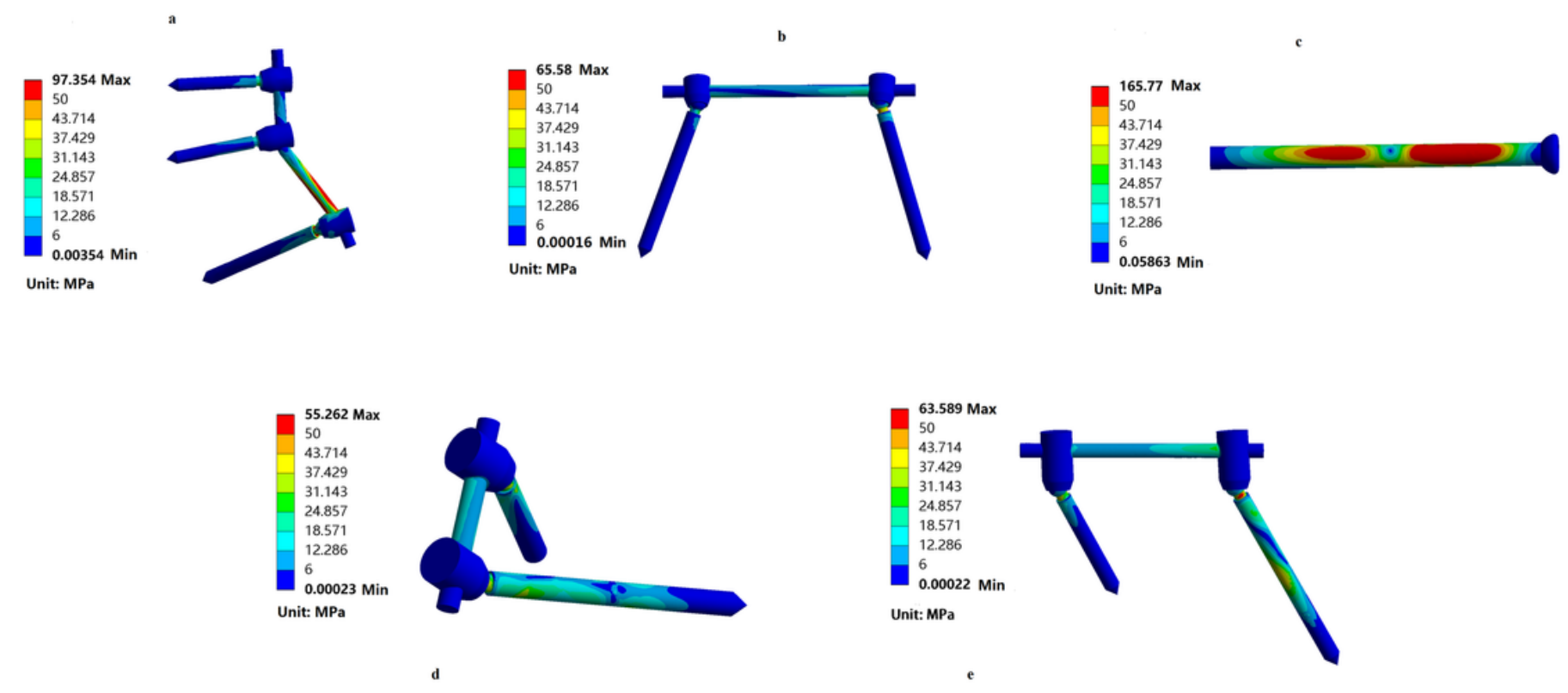

\section{Figure 3}

Stress distribution of the five implants under standing condition. a. LPF group, the maximum equivalent stress was located at the connecting rod near the iliac screw head. b. TIFI group, the maximum equivalent stress was located at the junction of the right iliac screw head and shaft. c. SIS group, the maximum equivalent stress was located at the middle of the screw. d. S2Al-S1 group, the maximum equivalent stress was located at the junction of the S1 pedicle screw head and shaft. e. S2AI-CS1 group, the maximum equivalent stress was located at junction of the S2AI screw head and shaft. 

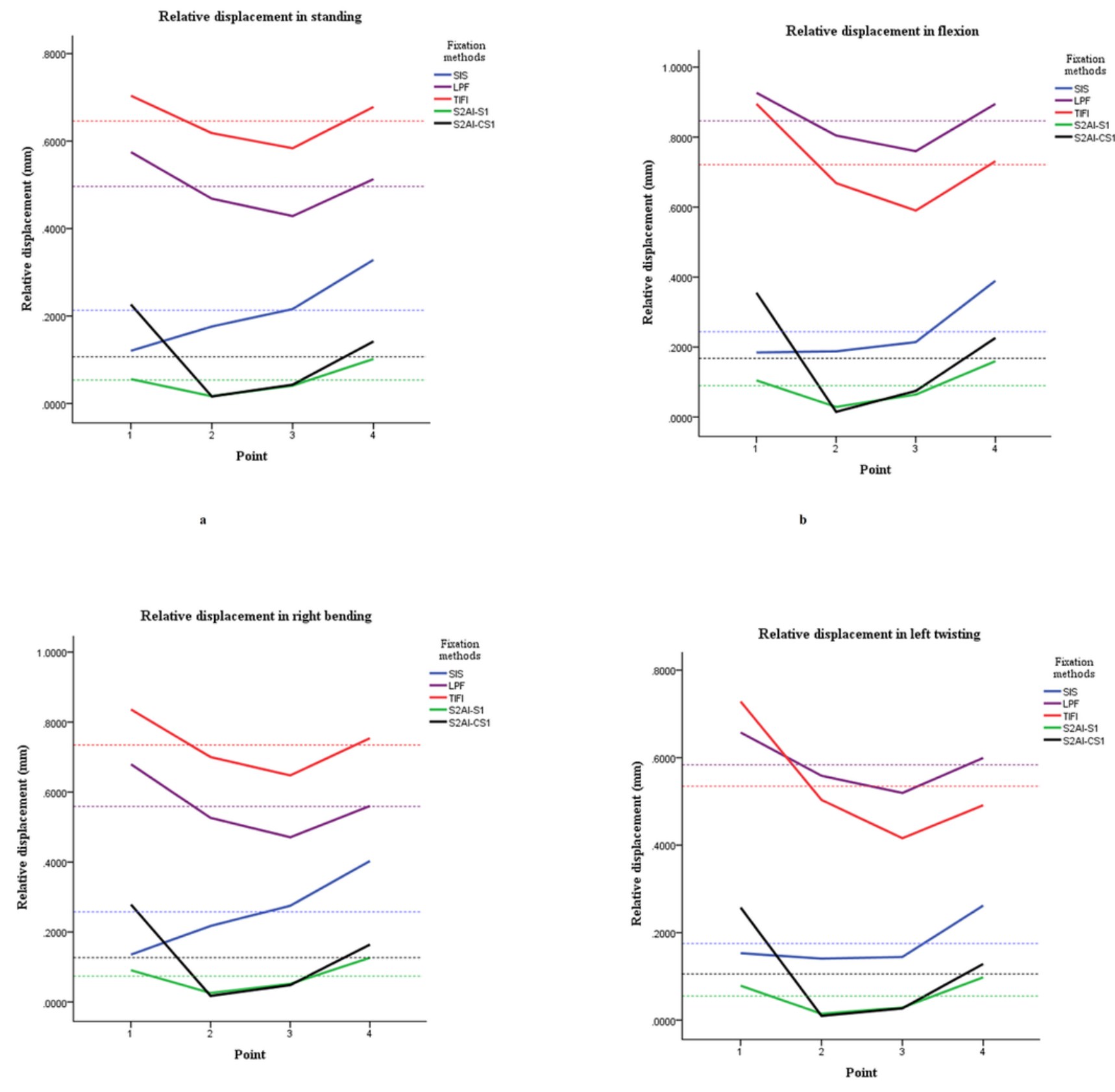

c

d

\section{Figure 4}

The relative displacement distribution diagrams in four different conditions. The dotted line of each color corresponds to the solid line of each color. The dotted line represents the average relative displacement. a .The relative displacement in standing . b. The relative displacement in flexion. $c$. The relative displacement in right bending. $d$. The relative displacement in left twisting .

\section{Supplementary Files}


This is a list of supplementary files associated with this preprint. Click to download.

- Additionalfile1.pdf

- Additionalfile2.pdf

- Additionalfile3.pdf

- Additionalfile4.pdf

- Additionalfile5.pdf 